\title{
Identificación y conteo de parásitos gastrointestinales en ovinos dorper, en Atlapexco, Hidalgo, México
}

\section{Identification and counting of gastrointestinal parsasites in dorper sheep, in Atlapexco, Hidalgo, Mexico}

\begin{abstract}
José de Jesús Pérez-Bautista ${ }^{1}$ (D) Ricardo Martínez-Martínez2(1D) Martin Hernández-Mogica ${ }^{3}$ (iD) María Teresa González-Lemus 3 [D Isaí de Jesús Austria-Hernández (D) Sergio Iban Mendoza-Pedroza

${ }^{1}$ Estudiante de Doctorado en Ciencias Agropecuarias, Universidad Autónoma de San Luis Potosí. Carretera San Luis - Matehuala Km. 14.5, Ejido Palma de la Cruz, CP. 78321 Soledad de Graciano Sánchez, San Luis Potosí, México.

${ }^{2}$ Universidad de Guadalajara, Centro Universitario la Costa Sur. Departamento de Producción Agrícola. Av. Independencia Nacional 151, Centro, CP. 48900. Autlán de Navarro, Jalisco, México.

${ }^{3}$ Tecnológico de México, Campus Huejutla de la Ingeniería en Agronomía. Carr. Huejutla - Chalahuiyapa $s / n$, Centro, CP. 43000. Huejutla, Hidalgo, México.

${ }^{4}$ Colegio de Postgraduados Campus Montecillo. Carretera México-Texcoco, km 36.5 Montecillo, CP. 56230. Municipio de Texcoco, Estado de México, México.

*Autor de correspondencia: ricardo.mmartinez@academicos.udg.mx
\end{abstract}

\section{Nota científica}

Recibida: 22 de febrero 2021 Aceptada: 11 de octubre 2021

Como citar: Pérez-Bautista JJ, MartínezMartínez R, Hernández-Mogica M, González-Lemus MT, Austria-Hernández IJ, Mendoza-Pedroza SI (2021) Identificación y conteo de parásitos gastrointestinales en ovinos dorper, en Atlapexco, Hidalgo, México. Ecosistemas y Recursos Agropecuarios Núm. Esp. II: e2873. DOI: 10.19136/era.a8nll.2873
RESUMEN. El objetivo del trabajo fue identificar y contar parásitos gastrointestinales (PGI) en un centro de producción ovina en Atlapexco, Se realizó un estudio coproparasitoscópico mediante la técnica de McMaster, a 42 ovinos Dorper, en septiembre a noviembre de 2017. Se aplicó una estadística descriptiva a las variables evaluadas (identificación y conteo de $\mathrm{PGl}$ por edad de los animales) con StatSoft System ${ }^{\circledR} \vee 7.1$. Los géneros de helmintos encontrados, en orden de mayor a menor proporción tanto en cada edad y mes fueron: Trichostrongylus spp., Trichurys spp. y Moniezia spp. (este último es un cestodo). Se encontró que los ovinos mayores a 12 meses presentaron mayor carga de parásitos (50.00-30000.00 con media 4742.8 PGI), mientras que los ovinos menores a 6 meses de edad tuvieron: 100.00-33000.00 con media 3800.0 PGl y los de 6-11 meses tuvieron: 50.00-9300.00 y media 2561.1 PGI. Esto permite tomar decisiones para reducir parasitosis en ovinos Dorper.

Palabras clave: Endoparásitos, especies, McMster, ovinos de pelo, sanidad.

ABSTRACT. The objective of the work was to identify and count gastrointestinal parasites (PGI) in a sheep production center in Atlapexco, A coproparasitoscopic study was performed using the McMaster technique, to 42 Dorper sheep, in September to November 2017. Descriptive statistics were applied to the evaluated variables (identification and PGI count by age of the animals) with StatSoft System ${ }^{\circledR} \vee 7.1$. The helminth genera found, in order from highest to lowest proportion both in each age and month were Trichostrongylus spp., Trichurys spp. and Moniezia spp. (the latter is a cestode). It was found that sheep older than 12 months had a higher parasite load (50.00-30000.00 with mean 4742.8 PGI), while sheep younger than 6 months of age had: 100.00-33000.00 with mean 3800.0 PGI and those of 6-11 months had: $50.00-9300.00$ and mean 2561.1 PGI. This allows making decisions to reduce parasitosis in Dorper sheep.

Key words: Endoparasites, species, McMster, hair sheep, health. 


\section{INTRODUCCIÓN}

En México, en los recientes años, la ovinocultura ha sido una actividad ganadera que va en aumento con un inventario de 8.7 millones de cabezas de ovinos en producción, principalmente por la calidad de la carne de estos animales para consumo como fuente de proteína animal para la población (SIAP 2019). Sin embargo, como en todo sistema de producción animal existen problemáticas y entre las más importantes se encuentra la sanidad y la alimentación inadecuada (Martínez-Martínez et al. 2019). En los sistemas de producción extensiva de ovinos, una de las principales enfermedades identificadas es la coccidiosis, y los vermes o parásitos gastrointestinales (PGI) (Sepúlveda-Vázquez et al. 2018). Esta problémica se remarca más donde los ovinos están en pastoreo y donde el clima y las condiciones ambientales son propicias para la proliferación de los PGI (Hernández et al. 2007). Entre los PGI que afectan a los ovinos de pelo están Trichostrongylus spp. y Trichurys spp., los cuales causan infecciones, repercuten en pobre ganancia de peso del animal, condición corporal baja y anemia, entre otras y por consecuencia, pérdidas económicas para el productor (Hernández-Alvarado et al. 2018). Para saber la carga parasitaria en ovinos existen técnicas sencillas, como la de MacMaster, método de concentración por flotación con solución saturada de azúcar (Hansen y Perry 1994, Rodríguez et al. 1994). Por ello es de suma importancia identificar si los animales aún después de desparasitarlos con compuestos químicos son susceptibles de una parasitosis y en qué proporción, para de esta forma aplicar soluciones a esta problemática y reducir las cargas de PGI en los ovinos de engorda. En la Huasteca Hidalguense, como en muchas otras regiones, esta problemática se presenta en los sistemas extensivos de producción ovina, por ello es necesario realizar estudios aplicar pruebas de detección y cuantificación parasitaria, en los centros de producción para poder dar una solución a esta problemática. Por lo anterior, el objetivo de la presente investigación fue identificar $y$ contar la población de parásitos gastrointestinales presentes en ovinos de engorda de la raza Dorper pastoreando Cynodon plectostachuys, en el municipio de Atlapexco de la Huasteca Hidalguense, Hidalgo.

\section{MATERIALES Y MÉTODOS}

\section{Localización del área de estudio}

La investigación se realizó en el rancho Teocuatitla, localizado en el municipio de Atlapexco, en la región huasteca del estado de Hidalgo, localizada a $21^{\circ} 00^{\prime}$ y $15^{\circ} 00^{\prime} \mathrm{LN}$, y $98^{\circ} 30^{\prime}$ y $20^{\circ}$ 00' LO, a $300 \mathrm{msnm}$ (INEGI 2015). La temperatura promedio es de $22{ }^{\circ} \mathrm{C}$, con precipitación anual de 1800 mm (CONAGUA 2015). El clima es cálido extremoso (García 2004).

\section{Animales y Manejo}

La investigación tuvo una duración de tres meses, de septiembre a noviembre de 2017. Se analizaron las heces de 42 ovinos de la raza Dorper de diferentes edades fisiológicas (menores de 6 meses, 6-11 meses y más de 12 meses), los cuales estuvieron en pastoreo extensivo con Cynodon plectostachuys, los muestreos se realizaron cada 30 días considerando que el ciclo biológico de los PGI ocurre en un mínimo de 21 días después de la ingesta por el animal (Suárez 2007). Se pesaron todos los animales en una báscula romana y con base en el peso vivo individual, se les administró el tratamiento antihelmíntico. Se desparasitaron dos veces, en los meses de julio y octubre, de acuerdo a los registros del productor, se les administró Ivermectina (Silvermec ${ }^{\circledR}$ ) a una dosis de $1 \mathrm{mg}$ por $\mathrm{kg}$ de peso corporal por inyección subcutánea.

\section{Obtención y procedimientos de muestra de heces}

La toma de muestra de heces se realizó antes del pastoreo de los ovinos (7:00 a 9:00 am), se colectaron $100 \mathrm{~g}$ de heces aproximadamente, directamente del recto del animal, para ello se utilizaron bolsas de plástico debidamente identificadas, se tomaron tres diferentes muestras durante toda la evaluación. Las muestras de heces, fueron llevadas al Laboratorio de Salud Animal A.C. el cual se localiza 
en el municipio de Huejutla de Reyes, del estado de Hidalgo.

Para la técnica de McMaster se tomaron 2 $\mathrm{g}$ de heces y se disolvieron en una solución azucarada sobresaturada, mezclándose hasta lograr la homogenización de las muestras. Posteriormente, con la mezcla se llenaron las cámaras McMaster y se colocaron en un microscopio compuesto (Amscope B120b Siedentopf) para ser observadas con el objetivo 10X, y determinar los niveles de infestación de los parásitos gastrointestinales por animal. Los análisis coprológicos, se procesaron individualmente, el mismo día de la obtención de las heces se realizó el conteo total de huevos por gramo de heces y se calculó multiplicando el número de huevos encontrados en ambas cámaras del dispositivo de McMaster por 50 de acuerdo a la técnica descrita por Rodríguez et al. (1994).

\section{Identificación de los Parásitos Gastrointestinales (PGI)}

Se identificaron los géneros de PGI, aislando las larvas $L_{3}$ mediante la técnica de coprocultivo. Las larvas $L_{3}$ obtenidas fueron identificadas por sus características morfológicas de tamaño, forma, número de células intestinales, y estructuras del extremo anterior y posterior (Liébano 2004).

\section{Análisis estadístico}

El análisis de los datos, identificación y conteo de huevos de parásitos totales en heces, edad de los animales y mes de muestreo, fue descriptivo utilizando la media y se usó el software STATISTICA ${ }^{\circledR}$ V 7.1 (StatSoft 2005).

\section{RESULTADOS Y DISCUSIÓN}

Los géneros de los parásitos gastrointestinales $\left(L_{3}\right)$, encontrados en los ovinos Dorper fueron tres en porcentaje de mayor a menor: Trichostrongylus spp. con $99.2 \%$, Trichurys spp. con $30 \%$ y Moniezia spp. con $0.16 \%$, respectivamente (Figura 1). El porcentaje promedio general de ovinos que fueron positivos a cargas parasitarias por PGI fue de $54.76 \%$. En donde los ovinos de 6-11 meses presentaron el mayor número de $\mathrm{PGI}$ con $61.53 \%$ a diferencia de los ovinos mayores a 12 meses que presentaron un $50 \%$ y con ovinos menores de 6 meses de $53.33 \%$, de acuerdo con la prueba de McMaster (Tabla 1). Los ovinos de un año en adelante fueron los más afectados con cargas parasitarias altas en promedio de 50.00-30 000.00 y media de 4742.8 , mientras que los ovinos menores a 6 meses de edad tuvieron en promedio cargas de parásitos de 100.00-33 000.00 con media de 3800.0 y los animales de 6-11 meses mostraron 50.00-9 300.00 con media de 2561.1 de parásitos gastrointestinales (Tabla 2). Esto indica que a mayor edad los animales son más susceptibles a parasitarse con nematodos, posiblemente a que estos ovinos están en constante contacto con el pasto, que representa la principal fuente de contagio, donde las larvas $\left(L_{3}\right)$ de los $P G I$ habitan y al consumirlas el animal se infecta (Marín et al. 2020). De acuerdo con DíazRivera et al. (2000) en el Estado de Veracruz, los ovinos que se encuentran en praderas libres son más propensos a la adquisición de parásitos gastrointestinales. Situación similar se reporta con ovinos en pastoreo en Cuetzala del Progreso, Guerrero (Hernández et al. 2007).

Las $L_{3}$ de Trichostrongylus spp., de ovinos con edad mayor a 12 meses produjeron los conteos más altos (6 $620.00 \pm 13$ 077.92), pero con ausencia de Trichurys spp. $(0.00 \pm 0.00)$ y valor bajo de Moniezia spp. (50.00 \pm 0.00 cestodos) en comparación con las otras edades de los ovinos. En los ovinos con edad menor a 6 meses se encontraron en promedio: $\mathrm{L}_{3}$ de Trichostrongylus spp. $4712.50 \pm 11$ 440.83, Trichurys spp. $150.00 \pm 70.71$ y Moniezia spp. 0.00 \pm 0.00 , es decir, hubo ausencia de cestodos. Los menores conteos de parásitos fueron encontrados en ovinos de 6-11 meses: $L_{3}$ de Trichostrongylus spp., $3257.14 \pm 39$ 97.02, Trichurys spp. $200.00 \pm$ 0.00 y Moniezia spp., $50.00 \pm 0.00$ cestodos (Tabla 3). Esto concuerda y se corrobora con lo reportado por algunos autores, donde analizaron y determinaron la presencia de estos géneros de PGI en ovinos de pelo en México (Salgado-Moreno et al. 2017, Rivero-Pérez et al. 2019) y otras partes del mundo (Zapata et al. 2016, Sánchez et al. 2019) encontrándose principalmente los géneros Trichostrongylus 


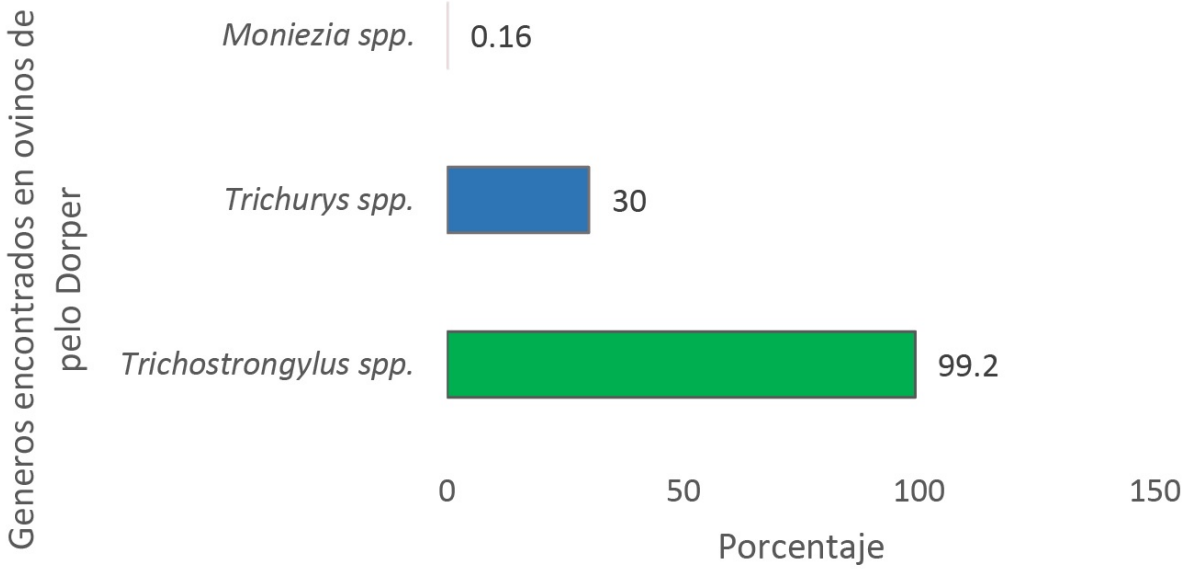

Figura 1. Prevalencia de los géneros de $P G I\left(L_{3}\right)$ en ovinos Dorper de Atlapexco, Hidalgo.

Tabla 1. Porcentaje de ovinos en pastoreos extensivos a positivos y negativos de Parásitos gastrointestinales.

\begin{tabular}{cccccc}
\hline \multirow{2}{*}{$\begin{array}{c}\text { Edad de los } \\
\text { ovinos (meses) }\end{array}$} & \multicolumn{5}{c}{ Ovinos } \\
\cline { 3 - 6 } & $N$ & \multicolumn{2}{c}{ Positivos } & Negativos \\
\cline { 3 - 6 } \cline { 3 - 5 } & & No. \% & No. \% & \\
\hline$<6$ & 15 & 8 & 53.33 & 7 & 46.66 \\
$6-11$ & 13 & 8 & 61.53 & 5 & 38.46 \\
$>12$ & 14 & 7 & 50.00 & 7 & 50.00 \\
Total & 42 & 23 & 54.76 & 19 & 45.23 \\
\hline
\end{tabular}

Tabla 2. Promedios de carga parasitaria (hpg) en ovinos Dorper a diferente edad, del Rancho Tiocuatitla en Atlapexco, Hidalgo.

\begin{tabular}{cccc}
\hline $\begin{array}{c}\text { Edad de ovinos } \\
\text { (meses) }\end{array}$ & $\begin{array}{c}\text { Ovinos } \\
\text { positivos }\end{array}$ & $\begin{array}{c}\text { Carga parasitaria } \\
\text { Media de hpg }\end{array}$ & Min - Max \\
\hline$<6$ & 8 & 3800.0 & $100.00-33000.00$ \\
$6-11$ & 8 & 2561.1 & $50.00-9300.00$ \\
$>12$ & 7 & 4742.8 & $50.00-30000.00$ \\
Total & 23 & 3625.00 & $66.66-24100.00$ \\
\hline
\end{tabular}

hpg: huevos de nematodos por gramo de heces.

spp., Haemonchus contortus, Trichostrongylus colubriformis y Cooperia curticei (Morales et al. 2006, López et al. 2013). Los resultados encontrados, indican que a mayor edad los ovinos son más parasitados, lo que concuerda con lo reportado por DíazAnaya et al. (2017), quienes reportaron que en ovejas mayores de 24 meses de edad presentan la mayor carga parasitaria.

Los géneros de PGI encontrados en ovinos Dorper en pastoreo extensivo del mes de septiembre fueron Trichostrongylus spp., más de 1000 larvas y en menor cantidad se encontró Trichurys spp., 300 larvas y ausencia de Moniezia spp. En octubre, Tri- chostrongylus spp., 100 larvas, Trichurys spp., ausencia de larvas y Moniezia spp., 100 larvas de cestodos. En noviembre se observaron, Trichostrongylus spp., más de 1000 larvas, Trichurys spp., 300 larvas y Moniezia spp., 50 larvas de cestodos. Esto concuerda con estudios realizados en Córdoba, Colombia, donde el género Trichostrongylus spp. fue uno de los nematodos predominantes en ovinos en pastoreo y en menor proporción Moniezia spp. (EnsunchoHoyos et al. 2014, Pulido-Medellín et al. 2014). En los meses evaluados, la presencia de parásitos en ovinos fue variable (en promedio, fue menor en octubre), pero se mantuvo la relación de mayor número 
Tabla 3. Número de parásitos con relación a la edad de los animales.

\begin{tabular}{cccccc}
\hline $\begin{array}{c}\text { Edad de ovinos } \\
\text { (meses) }\end{array}$ & Animales & \multicolumn{3}{c}{ Genero de Nematodos Gastrointestinales identificados } \\
positivos & Trichostrongylus spp & Trichurys spp & Moniezia spp & Total \\
\hline$<6$ & 8 & $4712.50 \pm 11440.83$ & $150.00 \pm 70.71$ & $0.00 \pm 0.00$ & 38000 \\
$6-11$ & 8 & $3257.14 \pm 3997.02$ & $200.00 \pm 0.00$ & $50.00 \pm 0.00$ & 23050 \\
$>12$ & 7 & $6620.00 \pm 13077.92$ & $0.00 \pm 0.00$ & $50.00 \pm 0.00$ & 33200 \\
\hline
\end{tabular}

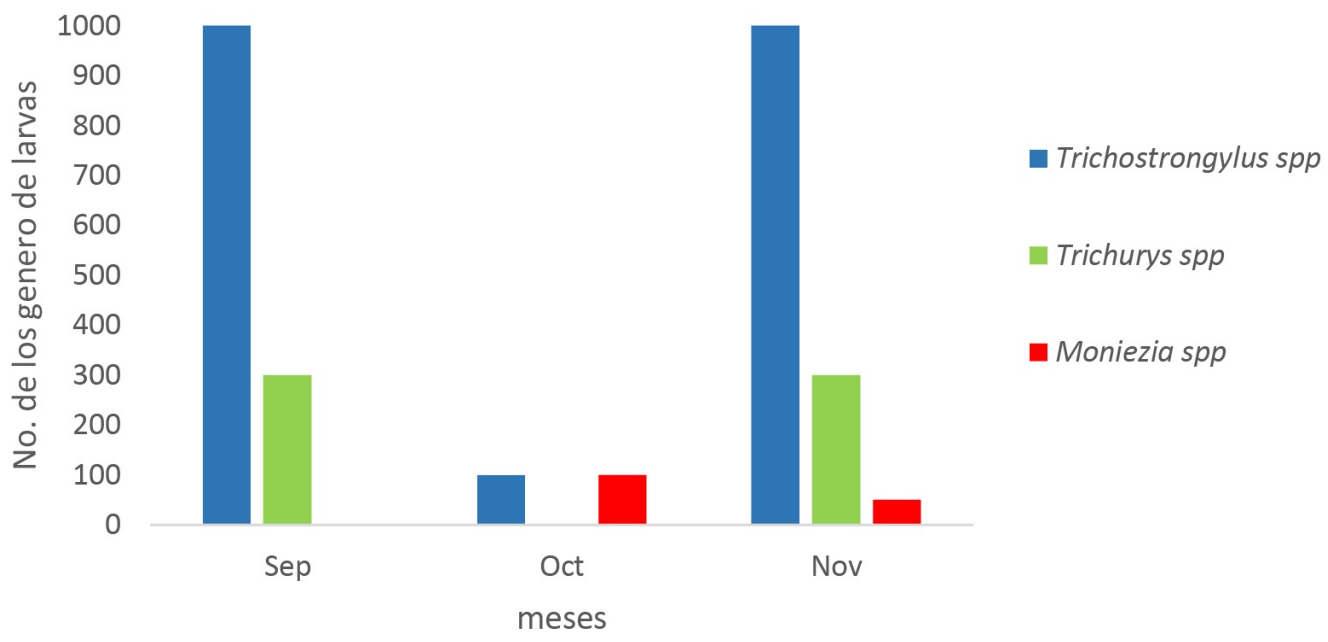

Figura 2. Frecuencia de los géneros de PGI encontrados en ovinos Dorper en pastoreo extensivo del mes de septiembre a noviembre, en Atlapexco, Hidalgo.

promedio de parásitos del género Trichostrongylus spp., seguido por Trichurys spp., y el menor número lo produjo Moniezia spp. Figura 2.

Una alternativa para un estudio posterior es reducir cargas parasitarias empleando metabolitos secundarios que contienen algunas leguminosas forrajeras, que afectan los diversos procesos biológicos de los nematodos gastrointestinales de rumiantes, y por consecuencia se reducen las cargas parasitarias en estos animales (Sepúlveda-Vázquez et al. 2018).

Se identificaron los géneros Trichostrongylus spp., Trichurys spp. y Moniezia spp. y la carga parasitaria total, aumentó con la edad del animal, con menor presencia en los meses de septiembre y noviembre.

\section{AGRADECIMIENTOS}

A los colaboradores de esta investigación, los cuales están en diversas instituciones como la Universidad Politécnica de Huejutla, Instituto Tecnológico de Huejutla, Universidad de Guadalajara y el Colegio de Postgraduados. Además de las facilidades prestadas en el rancho Teocuatitla en Atlapexco, Hidalgo.

\section{LITERATURA CITADA}

CONAGUA (2015) Comisión Nacional del Agua. Reporte de climas de México http://smn.cna.gob.mx/climatologia/ analisis/reporte/RC-Junio15.pdf. Fecha de consulta: 5 de julio de 2015.

Díaz-Anaya AM, Chavarro-Tulcán GI, Pulido-Medellín, MO, García-Corredor D, Vargas-Avella JC (2017) Estudio coproparasitológico en ovinos al pastoreo en Boyacá, Colombia. Revista de Salud Animal 39: 1-8.

Díaz-Rivera P, Torres-Hernández Glafiro, Osorio-Arce MM, Pérez-Hernández P, Pulido-Albores AR, BecerrilPérez CM, Herrera-Haro JG (2000) Resistencia a parásitos gastrointestinales en ovinos florida, pelibuey y 
sus cruzas en el trópico mexicano. Agrociencia 34: 13-20.

Ensuncho-Hoyos C, Castellano-Coronado A, Maza-Ángulo L, Bustamante-Yánez M, Vergara-Garay O (2014) Prevalencia y grado de infección de nematodos gastrointestinales en ovinos de pelo en pastoreo de cuatro municipios de Córdoba, Colombia. Revista Científica 24: 414-420.

García E (2004) Modificaciones al sistema de clasificación climática de Köppen. Universidad Nacional Autónoma de México, Instituto de Geografía. México. 91p.

Hansen J, Perry B (1994) The epidemiology, diagnosis and control of helminth parasites of ruminants. A Handbook, 2nd ed. International Laboratory for Research on Animal Diseases. Nairobi, Kenya. 171p.

Hernández RS, Segura GI, Pérez OJ, Almazán VMT (2007) Prevalencia de nematodos gastrointestinales en ovinos en pastoreo en la parte alta del Mpio. De Cuetzala del Progreso, Guerrero-México. Revista Electrónica de Veterinaria 8: 1-7.

Hernández-Alvarado J, Zaragoza-Bastida A, López-Rodríguez G, Peláez-Acero A, Olmedo-Juárez A, RiveroPérez N (2018) Actividad antibacteriana y sobre nematodos gastrointestinales de metabolitos secundarios vegetales: enfoque en Medicina Veterinaria. Abanico veterinario 8: 14-27.

INEGI (2015) Principales resultados por localidad 2010 (ITR). Instituto Nacional de Estadística y Geografía. http://www.inegi.org.mx/sistemas/consulta_resultados/iter2010.aspx. Fecha de consulta 1 de julio 2017.

Liébano HE (2004) Identificación morfométrica de larvas infectantes de nematodos gastrointestinales y pulmonares en rumiantes domésticos de México. En: Diagnóstico y control de los nematodos gastrointestinales de los rumiantes en México. Instituto Nacional de Investigaciones Forestales Agrícolas y Pecuarias. Centro de Investigación Disciplinaria en Parasitología Veterinaria (CENID-PAVET). Jiutepec, Morelos, México. pp: 28-29.

López ROA, González GR, Osorio AMM, Aranda IE, Díaz RP (2013) Cargas y especies prevalentes de nematodos gastrointestinales en ovinos de pelo destinados al abasto. Revista mexicana de ciencias pecuarias 4: 223-234.

Marín MSC, Cortez RA, Díaz BMB (2020) Presencia de parásitos gastrointestinales en ovinos de la Estación Experimental Choquenaira, municipio de Viacha, La Paz. Revista Estudiantil AGRO-VET 4: 504-509.

Martínez-Martínez R, Vicente-Pérez R, García-Flores EO, Grifaldo-Alcántara PF, Vargas-Madriz H, Pérez-Bautista JJ, Soriano-Robles R, García-Alonso F (2019) Efecto de extracto de taninos condensados in vitro en huevos de nematodos gastrointestinales de ovinos. Revista Mexicana de Agroecosistemas. 6: 16-18.

Morales G, Pino LA, Sandoval E (2006) La estrongilosis digestiva de los ovinos a pastoreo en Venezuela. REDVET. Revista Electrónica de Veterinaria 7: 1-15.

Pulido-Medellín MO, García-Corredor D, Díaz-Anaya A, Andrade-Becerra R (2014) Pesquisa de parásitos gastrointestinales en pequeñas explotaciones ovinas del municipio de Toca, Colombia. Revista Salud Animal 36: 65-69.

Rivero-Pérez N, Jaramillo Colmenero A, Peláez-Acero A, Rivas-Jacobo M, Ballesteros-Rodea G, ZaragozaBastida A (2019) Actividad antihelmíntica de la vaina de Leucaena leucocephala sobre nematodos gastrointestinales de ovinos (in vitro). Abanico Veterinario 9: 1-9.

Rodríguez VRI, Domínguez AJL, Cob GLA (1994) Técnicas diagnósticas de parasitología veterinaria. Universidad Autónoma de Yucatán. Mérida, Yucatán, México. 236p. 
Salgado-Moreno S, Carrillo-Díaz F, Escalera-Valente F, Delgado-Camarena C (2017) Pruebas para identificar ovinos resistentes a parásitos gastrointestinales en San Pedro Lagunillas Nayarit. Abanico veterinario 7: 63-71.

Sánchez AL, Bell, Washington, Ponzoni, Raúl W (2019) Consideraciones acerca de la precisión necesaria en el conteo de huevos de parásitos internos en heces de ovinos. Agrociencia (Uruguay) 23: 76-79.

Sepúlveda-Vázquez J, Torres-Acosta JF, Sandoval-Castro CA, Martínez-Puc JF, Chan-Pérez JI (2018) La importancia de los metabolitos secundarios en el control de nematodos gastrointestinales en ovinos con énfasis en Yucatán, México. Journal of the Selva Andina Animal Science 5: 79-95.

SIAP (2019) Resumen nacional: producción, precio, valor, animales sacrificados y peso. Servicio de Información Agroalimentaria y Pesquera. http://www.siap.gob. Fecha de consulta: 3 enero 2020.

StatSoft (2005) Statistica data analysis software system. Versión 7.1. wwwstatsoft.com. Fecha de consulta: 5 de enero 2021.

Suárez VH (2007) Sistemática y bionomía de los principales nematodos de los lanares. In: Inta (ed) Enfermedades parasitarias de los ovinos y otros rumiantes menores. Publicación técnica No. 70. Anguil, Argetina. pp: 15-32.

Zapata SR, Velásquez VR, Herrera OLV, Ríos OL, Polanco EDN (2016) Prevalencia de Nematodos Gastrointestinales en sistemas de producción ovina y caprina bajo confinamiento, semiconfinamiento y pastoreo en Municipios de Antioquia, Colombia. Revista de Investigaciones Veterinarias del Perú 27: 344-354. 